\title{
Does contextual change determine long-term forgetting?
}

\author{
PASCALE GISQUET-VERRIER and TATIANA ALEXINSKY \\ C.N.R.S., Gif sur Yvette, France
}

\begin{abstract}
A series of experiments were run to test the hypothesis that "spontaneous forgetting" could result from subtle contextual changes. The first experiment demonstrated that when SpragueDawley male rats are trained in a runway alley with a food reinforcer, retention performance is dramatically affected by a change in the pattern of the walls of the training apparatus when testing takes place 1, 3, or 5 days following training and not after 1 week. Experiment 2 demonstrated that this performance deficit cannot be alleviated by any of the three selected cuing treatments, whereas "spontaneous forgetting" (resulting from a training-to-test interval of 14 days) can be. These data indicate that the detrimental effect of contextual change reduces over time, and that such an effect cannot be interpreted in terms of retrieval failure. These results lend strong support to the argument that the disruptive contextual change effect is basically different from the disruptive effect that results from an extended training-to-test interval.
\end{abstract}

It is now well established, in both human and animal studies, that any alteration between training and testing contexts may induce performance disruption at testing (see Spear, 1978, and Riccio, Richardson, \& Ebner, 1984, for reviews). With human subjects, slight modifications of minor environmental features, such as changes in the color of the print of words (Postman, 1971), a change in sensorial modality used for the presentation of a verbal item-visual or acoustical (Smith, Glenburg, \& Bjork, 1978), changes made in the experimental room (Keppel, 1972), all lead to a deterioration in retention of verbal materials. Similar evidence comes from studies with animals, which exhibit poor performance when the environmental context is modified between training and testing. Such alterations of the context may concern external stimuli, change of experimental room (Gordon, McCracken, Dess-Beech, \& Mowrer, 1981), slight modifications of the training apparatus (Perkins \& Weyant, 1958; W. R. McAllister \& D. E. McAllister, 1963), or internal stimuli, such as changes in the internal state of the animal following injections of drugs (cf. state dependency phenomenon; Overton, 1964) and changes in the time of the day when the experiment takes place (Holloway \& Wansley, 1973).

All these studies point out the importance of maintaining similarity between training and testing conditions in determining levels of retention performance, as emphasized by Tulving and Thomson (1973) in their encoding specificity theory. Spear $(1973,1978)$ has provided a theoretical framework for the critical role of context in the mechanisms of forgetting and memory retrieval. According to Spear, the differences between the context accompanying acquisition and the context at the time of testing

\footnotetext{
The authors' mailing address is: Departement de Psychophysiologie Laboratoire de Physiologie Nerveuse, C.N.R.S., 91190 Gif sur Yvette, France.
}

are the major determinants of forgetting. He suggests that two classes of experimental results could provide evidence consistent with this position: "Forgetting is either increased by contextual change or is decreased by eliminating contextual change' (Spear, 1978, p. 52).

Spear indicates that the latter alternative could be addressed by means of pretest cuing procedures in which subjects are exposed to some elements of the training situation before testing. Such treatments can alleviate performance deficits resulting from various sources: experimentally induced amnesia (Sara, 1973), "spontaneous forgetting" (defined simply as a decrease in performance after a long training-to-test interval) (Deweer, Sara, \& Hars, 1980), and specific retention intervals, such as those that lead to the Kamin effect (Gisquet-Verrier, Dekeyne, \& Alexinsky, 1985; Klein \& Spear, 1970) or even a warm-up decrement, at the beginning of the retention test (Spear, Gordon, \& Martin, 1973).

The function of pretest cuing is often considered to be that of reinstating, at the time of the retention test, the internal context of the animal or of evoking the context present during the initial training. Thus, according to Spear, pretest cuing increases the similarity between training and testing situations, which should lead to an enhancement of the retrievability, at the time of testing, of previously stored information.

In discussing the possibility that forgetting might be increased after contextual changes had taken place between training and testing, Spear referred to a number of the experiments mentioned earlier in this introduction. In fact, those experiments do not really demonstrate increased forgetting after contextual change, but, rather, show detrimental effects of contextual changes on test performance.

More recently, Riccio (Riccio \& Ebner, 1981; Riccio et al., 1984) raised an important question concerning the compatibility of (1) the notion that forgetting is due to 
change in context and (2) the numerous demonstrations that have been made of the flattening of contextual generalization gradients as a function of time. Perkins and Weyant (1958) reported that rats trained in a runway alley and then tested in an alley with a different pattern on its walls exhibited poorer test performance than did rats trained and tested under identical conditions, when the retention test took place shortly after initial training ( $1 \mathrm{~min}$ ) but not when 1 week was interpolated between training and testing. These data were confirmed by Steinman (1967), who obtained the same results using 1 and 66 days. A disruptive effect on test performance, resulting from a contextual change made only shortly after initial training, has been shown to occur with both appetitive tasks (Perkins \& Weyant, 1958; Steinman, 1967) and aversively motivated tasks (Desiderato, Butler, $\&$ Meyer, 1966; W. R. McAllister \& D. E. McAllister, 1963; Pesselman \& Riccio, 1980; Richardson, Riccio, Guanowsky, \& Okonkwo, 1983; Seybert, Wilson, \& Archer, 1982).

The most important point of these experiments is that they demonstrate that the passage of time overcomes the detrimental effects of contextual changes on performance. This was emphasized by Riccio et al. (1984) in their recent review of the literature: "Discriminable stimuli, as indexed by performance shortly after acquisition, become more equivalent or interchangeable in controlling responding, after a delay" (p. 158). Here is the so-called "paradox, " first alluded to by Riccio and Ebner (1981), which emerged from the discrepancy between the model of forgetting proposed by Spear (1978) and the experimental data concerning the contextual change effect.

The paradox is: How can it be maintained that one of the major determinants of forgetting is based upon mismatching between training and testing context (both external and internal) when numerous experimental data indicate that contextual shifts leave performance unaffected after a long delay?

In our opinion, such a paradox may result from the fact that arguments coming from experiments using contextual change have been used to account for spontaneous forgetting, while, at the same time, it has never been clearly established whether or not the contextual change effect can be accounted for in terms of a retention deficit. Spear (1978) labeled the performance deficit resulting from contextual change as forgetting, according to his operational definition. Gordon et al. (1981) went even further and included the contextual change effect under the general heading of forgetting. Gordon et al. performed a series of experiments aimed at demonstrating that, during cuing, the cuing context is added to the previously activated training memory. Such an addition would enhance the similarity between test stimuli and stimuli represented in training-reactivated memory. For their demonstration, they used a disruption of performance induced by a contextual change in the same way as other kinds of memory deficits, such as spontaneous forgetting or experimentally induced amnesia, can be. Hence, they implicitly considered this disruption of performance as being due to retrieval failure.

The purpose of the present series of experiments was to explore further the contextual change effect, with a view to illuminating Riccio's paradox. The aim of the first experiment was to establish the contextual change effect and to determine its time course. The aim of the second experiment was mainly to examine to what extent the pretest cuing procedure, already shown to be effective in reducing spontaneous forgetting, might induce a similar facilitative effect after performance disruption resulting from a contextual change introduced shortly after training.

\section{EXPERIMENT 1}

As previously indicated, Perkins and Weyant (1958) have shown that modifications of the pattern on the walls (black/white) of a runway alley disturb test performance when the modifications are introduced during a retention test occcurring shortly after appetitive training $(1 \mathrm{~min})$ but not after a longer retention interval ( 7 days). The purpose of the first experiment was to replicate these findings and to investigate more precisely the time course of the contextual change effect.

\section{Method}

The general design of this experiment was as follows: Rats were trained in a runway alley and tested after one of four retention intervals $(1,3,5$, or 7 days), either in the same alley as the one used during training (Condition NoC, no change) or in one that differed in terms of the pattern on its walls (Condition $\mathrm{C}$, change). If the animals performed differently in the two test environments, they would be considered to be reacting to the contextual change as a function of the change in this incidental feature.

The experiment was run in four replications, each of which included all the experimental conditions.

Subjects. The subjects were 120 male Sprague-Dawley rats obtained from the Iffa-Credo rearing center; they weighed $200 \mathrm{~g}$ at the beginning of the experiment. They were housed, in pairs, in wire-mesh cages measuring $35 \times 20 \times 18 \mathrm{~cm}$. Eight days after the rats' arrival, the following food-deprivation schedule was introduced: the daily ration (given between 4 and 6 p.m. and at least 30 min after their daily training sessions) was gradually reduced over a period of 12 days from ad lib to $10 \mathrm{~g}$ of food. This ration was given on the 2 days preceding training. Under this regimen, with water available ad lib, the weights of the animals increased slightly during the experiment.

Apparatus. The training apparatus (described in detail by GisquetVerrier, 1981) consisted of two alleys $(150 \times 23 \times 32 \mathrm{~cm})$ with a 20-cm-long startbox, a 120-cm-long runway, a 28-cm-long goalbox, and 32-cm-high walls. Two side-opening perspex doors separated the three sections from each other. The total running time, automatically recorded, was the time that elapsed from the opening of the door to the breaking of the photoelectric-cell beam located $25 \mathrm{~cm}$ from the entrance of the goalbox. The two alleys were identical in every respect, except that the inside of one alley was painted flat white and the inside of the other alley was painted flat white with vertical black stripes. The two alleys were used alternately in the same location in an experimental room that was adjacent to the colony room. Each animal was manipulated by the same experimenter during pretraining, training, and testing, so that the 
only change the animals tested under the changed conditions were exposed to was the alteration in the pattern on the walls of the alley.

Procedure. The white alley was used for half of the animals, and the striped one was used for the other half. The rats were first habituated to the apparatus. On Day 1 , in groups of 6 , they were allowed to explore the apparatus, with the food-pellet reinforcement available in the goalbox for $20 \mathrm{~min}$. On Days 2 and 3, the rats learned, first in groups of 2 and then singly, to enter the goalbox, after which the door remained closed until they had eaten all the available pellets. Training trials were run on the following day; a single training session consisted of 12 reinforced trials with a 30 $\mathrm{sec}$ intertrial interval. During a trial, each subject was placed into the startbox for $30 \mathrm{sec}$; then the door was opened, and the running time to the goalbox was recorded. After eating one pellet, each animal was removed and replaced into the startbox. At the end of the training session, each subject was returned to its home cage and assigned to one of the eight groups on the basis of its performance during the last three acquisition trials.

Four training-to-test intervals (TTI) were used for studying retention: $1,3,5$, or 7 days. At the end of the TTI, each animal was given 12 retraining trials. Behavioral procedures were identical to those used during the original training except that, for half of the animals, retraining took place in the same training runway (nochange conditions, NoC), and for the other half, the retraining took place in the runway with the different wall pattern (change conditions, C) (NoC 1-day, $n=15$; C 1-day, $n=16$; NoC 3-day, $n=15$; C 3-day, $n=15$; NoC 5-day, $n=14$; C 5-day, $n=14$; NoC 7-day, $n=16$; C 7-day, $n=15$ ).

The 120 rats were assigned to one of the eight groups in a 2 (C or NoC) $\times 4$ (TTI $=1,3,5$, or 7 days) factorial design.

\section{Results}

To increase the homogeneity of variance, running times were subjected to a logarithmic transformation before statistical analysis.
Acquisition. No significant difference could be detected among the four separate replications $[F(3,112)<1]$. At the end of training, the animals had been divided into eight groups on the basis of their running times during training. The analysis of variance (groups $\times$ repetition) performed during the 12 training trials and among the eight groups submitted to different treatments and training-totest intervals indicated: (1) no difference between groups $[F(7,112)=0.34],(2)$ a significant effect of repetition $[F(11,112)=21.94, p<.001]$ demonstrating a significant decrease of running time in the course of learning, and (3) no interaction between groups and repetitions $[F(77,112)=0.86]$. Furthermore, no difference between groups could be detected in the response latencies on the last training trial $[F(7,112)=0.41]$.

Retention. As no difference could be detected between the animals trained in the white alley and those trained in the striped alley, the results were simply treated as a function of change (C) versus no change (NoC) between the training and testing phases (see Figure 1).

Within-group comparisons showed that when testing occurred 1,5 , or 7 days after initial training, the performance of the NoC animals in the first test trial was similar to their performance in the last training trial. Rats submitted to the 3-day TTI ran significantly faster during the first test trial than they had on the last training trials $[t(14)=4.46, p<.001]$.

Animals that had their retention test in a novel alley (C condition) exhibited longer response latencies on the first test trial than they had at the end of training, when testing occurred relatively soon after initial acquisition.

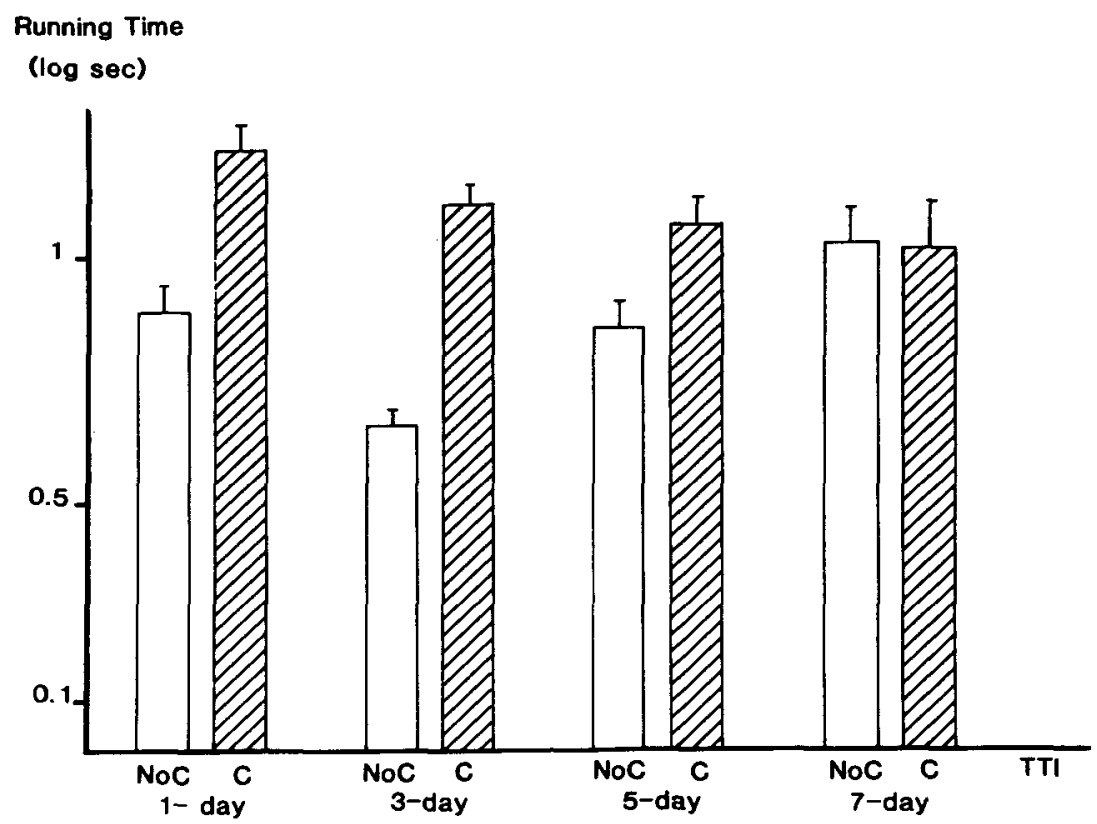

Figure 1. Mean $\log$ running time $( \pm S E M)$ on the first retention test trial for each of the TTI conditions (1, 3, 5, and 7 days). A score of 1 (10 sec to run the alley) corresponds to a speed of $14.8 \mathrm{~cm} / \mathrm{sec}$. Comparisons between animals tested in the training alley (NoC, white bars) and those tested in the other alley ( $C$, dotted bars) indicated that the contextual change induced a deleterious effect on performance 1,3 , and 5 days following training, but not after a retention interval of 7 days. 
Within-group comparisons showed this performance decrement to be significant for TTI 1-day $[t(15)=4.66$, $p<.001]$, TTI 3-day $[t(15)=5.12, p<.001]$, and TTI 5-day $[t(13)=2.60, p<.025]$.

For between-group comparisons, Figure 1 displays the mean running time, on the first test trial, for all animals tested at different TTIs. A two-way analysis of variance performed on these data indicated significant main effects of testing conditions $(\mathrm{C}$ or NoC) $[F(1,112)=33.93$, $p<.001]$ and of the training-to-test interval $[F(3,112)$ $=2.93, p=.036]$. A significant interaction between testing conditions and the length of retention interval $[F(3,112)=5.43, p<.001]$ revealed that the effect of change was a function of the length of the retention interval.

Comparison of $\mathrm{NoC}$ and $\mathrm{C}$ groups indicated significant effects of testing conditions after 1 day $[F(1,29)=18$, $p<.001], 3$ days $[F(1,28)=62.71, p<.001]$, and 5 days $[F(1,26)=6.71, p<.014]$. The disruptive effect of contextual change on performance was not restricted only to the first test trial; comparisons of treatment $x$ repetition indicated that this effect endured until the 4th to the 6th trials, depending on the TTI. After 7 days, the testing conditions no longer induced any difference between $\mathrm{C}$ and NoC animals $[F(1,29)=.011]$.

Despite the fact that the between-groups analyses performed on all the $\mathrm{C}$ groups do not reach an acceptable level of significance $[F(3,56)=1.92, p=.13]$, animals tested at 7 days performed better than those tested at 1 day $[F(1,29)=4.24, p=.046]$. In other words, the disrupting effect of contextual change decreases during the first week following initial acquisition.

Between-group analyses performed on the NoC groups indicated a significant effect of TTI on running times $[F(3,56)=6.90, p<.001]$. Further analyses showed that this effect was due mainly to an improvement in performance of the animals assigned to the 3-day TTI group. This group (NoC 3-day) exhibited significantly shorter running times than any of the other NoC groups $(.05<p<.01)$. These data strengthened the preceding within-group comparisons in indicating a spontaneous improvement in performance, or a "reminiscence" phenomenon, which has already been described in the literature (Huppert \& Deutsch, 1969; Madgsick, 1936). An explanation in terms of incidental modifications in motivational state is not likely, since the weights of all the animals regularly increased throughout the experiment.

In addition, in a comparison of the NoC 1-day and NoC 7-day groups no significant decrement in performance between Day 1 and Day 7 could be detected $[F(1,29)=$ 2.33, $p=.13]$. This indicated that, for animals trained and tested under the NoC conditions, the running response was well maintained 1 week after initial training.

\section{Discussion}

This experiment confirms and extends the previous findings of Perkins and Weyant (1958) and of Steinman (1967). The results indicate that a wall-pattern change be- tween training and testing situations may disturb test performance for several trials. However, the length of the delay between acquisition and test seems to be an important determinant of this effect: a contextual change significantly disrupted retention performance only when testing was run soon after training. The same modification encountered 7 days following training had no detectable effect. Furthermore, it appears that the performance of animals tested with changed conditions was better 1 week after initial acquisition than it was 1 day after acquisition, whereas the performance of the $\mathrm{NoC}$ control animals did not change between the 1- and 7-day test delays. The disruption induced by contextual change lessens over time and is no longer detectable before spontaneous forgetting occurs in NoC animals (see also Desiderato et al., 1966; Richardson et al., 1983).

In other words, alteration of a contextual stimulus has less and less effect on performance as the retention interval increases. These data obviously accentuate part of the paradox discussed above: how could a phenomenon that increases over time (forgetting) be accounted for by a mechanism whose effects decrease over time (contextual change effect)?

\section{EXPERIMENT 2}

The first experiment confirmed that some contextualcue change that occurs between training and testing situations leads to poor performance only when the retention test takes place soon after initial training. The principal aim of Experiment 2 was to examine whether the performance disruption resulting from a contextual change introduced soon after training could effectively be analyzed as a memory deficit, as suggested by Gordon et al. (1981). If the performance deficit seen after contextual change and after a long training-to-test interval have the same sources, that is, retrieval failure, then these deficits should respond similarly to pretest treatments.

Rats were trained as in the preceding experiment. Since the most important effect of contextual change had been obtained 1 day after training, and since pilot studies had indicated that spontaneous forgetting of the running response was observed after a 14-day TTI, the animals were tested for retention either 1 or 14 days after initial training.

Previous experiments had demonstrated that a pretest presentation of the experimental context alleviates forgetting (Deweer et al., 1980; Deweer \& Sara, 1984; GisquetVerrier et al., 1985). Those experiments as well as a pilot study with the runway had also demonstrated that such an effect was not due to a nonspecific arousal induced by manipulation of the animal. Therefore, in the present experiment, presentation of the experimental context was chosen as the pretest cuing treatment for alleviating performance disruption due to a 14-day retention interval. However, recent data had indicated that when the disruption occurred soon after training, such treatment could be less effective than when it occurred long after training 
(Gisquet-Verrier et al., 1985). For that reason, in a serious effort to reverse the detrimental effect of the contextual change, we studied several other effective reminders in addition to presentation of the experimental context. These reminders included presentation of the reinforcer (Koppenaal, Jagoda, \& Cruce, 1967) and placement in the startbox (Sara, 1973), both given 1 day following training. In addition, specificity of the cuing treatment after that particular interval was assessed by using a control group.

\section{Method}

The experiment was run over a period of several weeks. After training, each animal was assigned to one of 10 groups on the basis of acquisition performance.

Subjects. The subjects were 124 male albino rats, identical to those used in Experiment 1, and the same food-deprivation schedule was used.

Apparatus. The apparatus was the same as that employed in the first experiment. Cuing was delivered either in a cuing box $(23 \times 23 \times 36 \mathrm{~cm})$, which was similar to the startbox of the alley but differed in having a neutral color (beige), or in a wire-mesh cage that was similar to the animals' home cages.

Procedure. All rats received runway pretraining and training, according to the procedure of Experiment 1, with small pieces of hazelnuts as food reinforcer. At the end of training, the 124 rats were divided into 10 groups on the basis of their performance during acquisition. Six groups were submitted to the 1-day TTI, and the other four were submitted to the 14-day TTI.

As in Experiment 1, the rats were tested either in the training alley (NoC) or in a different alley (C). At the end of their respective retention interval, the noncued animals were removed from the home cage and carried in the experimenter's hand to the experimental room. They were then placed directly into the startbox, and the door was immediately opened. The animals were retrained for eight reinforced trials with a 30-sec intertrial interval.

The remaining animals were cued for $2.5 \mathrm{~min}$, then removed and returned to the home cage for $5 \mathrm{~min}$. The testing procedure was identical to that of the noncued animals.

For the 1-day TTI, the first two conditions, NoC $(n=11)$ and $\mathrm{C}(n=11)$, were the same as the corresponding conditions in Experiment 1 .

The four remaining groups received a pretest cue and were tested under changed conditions (C). For the first three cued groups, the cuing took place in the colony room. The cage group (Group Ca-C: $n=11$ ) was a control group; these animals were simply placed in a wire-mesh cage. The animals in the neutral startbox group (Group NSB-C: $n=10$ ) were placed in a cuing box that was identical to the startbox of the alleys, except that its walls were of a neutral beige color. The rats of the food group (Group F-C: $n=12$ ) were placed in the wire-mesh cage where they had access to hazelnuts. The animals in the experimental context group (Group EC: $n=10$ ) were cued in the experimental room, where they were placed in the wire-mesh cage, next to the initial training alley.

For the 14-day TTI, as previously, two groups were tested directly after the end of the retention interval, either with the training conditions (Group NoC: $n=14$ ) or with the changed conditions (Group C: $n=14$ ).

In the remaining groups, presentation of the experimental context (EC), already described, was used as a cuing treatment. The animals were tested either in nonchanged (Group EC-NoC: $n=15$ ) or in changed conditions (Group EC-C: $n=16$ ).

\section{Results}

Acquisition. After training, the animals were divided into 10 groups. An analysis of variance (group $\times$ repeti- tion) performed over the 12 training trials and among the 10 groups showed (1) no difference between groups $[F(9,114)=0.27],(2)$ a significant effect of repetition $[F(9,114)=19.63, p<.001]$, and (3) no interaction between groups and repetition, indicating that the animals had learned at the same rate. Furthermore, there was no difference between groups on the last training trial $[F(9,114)=0.17]$.

Retention. Performance relative to the one animal tested in the training alley 1 day following initial training (NoC 1-day) could be disrupted either by a contextual change introduced after the same retention interval (C 1day) $[F(1,20)=7.16, p=.014]$ or by a 14 -day trainingto-test interval (NoC 14-day) $[F(1,23)=12.36$, $p=.002$ ] (see Figure 2).

For the 1-day TTI, Figure 3 presents the mean log running time on the first test trial, for each of the six groups considered. Analyses of these data indicated a significant effect of change $[F(1,20)=7.16, p=.014]$, confirming the results of the first experiment: animals trained and tested in different contexts exhibited poorer retention performance $24 \mathrm{~h}$ after initial training than did animals trained and tested under identical conditions. This effect continued during the first six testing trials $[F(1,20)=$ $4.41, p<.05]$. Furthermore, the performance of the nonchanged groups also differed significantly from each of the cued, changed groups (statistical levels ranging from $p<.002$ to $p<.06$ ). Since, in addition, no significant differences could be detected between the five groups of animals tested under changed conditions but with different pretest treatments $[F(4,49)=.90]$, it appears that this performance deficit, induced by change, cannot be reduced by any of the proposed cuing treatments.

For the 14-day TTI, Figure 4 represents the mean log running times for each of the four groups on Test Trial 1 . Overall analysis of variance on these groups indicated that

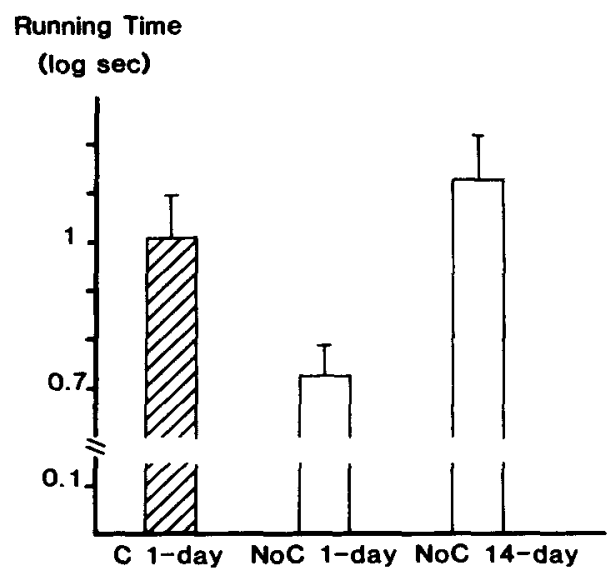

Figure 2. Mean log running time $( \pm S E M)$ on the first test trial for three particular treatment conditions. Note that, as compared with the performance of the NoC animals tested 1 day after training (NoC, 1-day), disruption can be obtained either by a contextual change made when testing was run 1 day after training (C, 1-day) or by a training-to-test interval of 14 days (NoC, 14-day). 


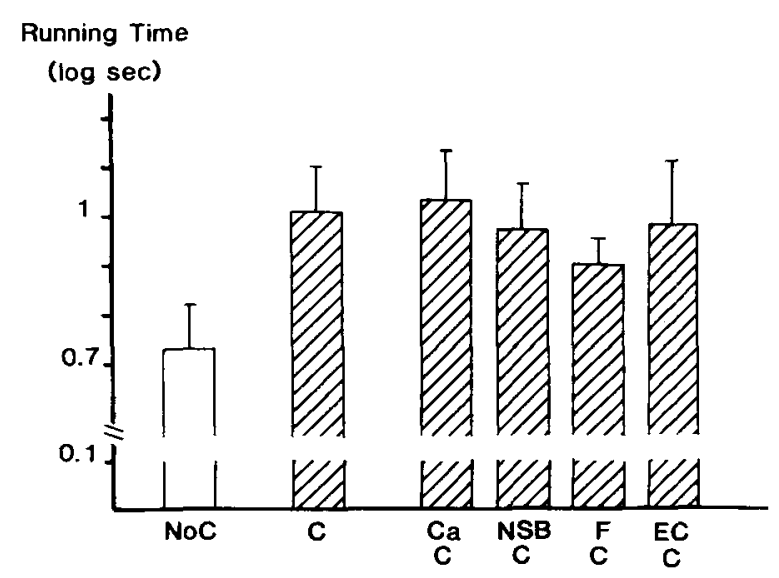

Figure 3. Mean log running time $( \pm S E M)$ during the first retention test trial for each of the groups tested after a 1-day TTI. Contextual change $(C)$ induced a disruption of performance relative to animals tested under training conditions (NoC). None of the $\mathrm{C}$ groups that were submitted to a cuing treatment: exposure to a neutral startbox (NSB), access to the food reinforcer (F), or exposure to the experimental context (EC) differed either from the control group of the cuing treatment (CA) or from the noncued changed group (C).

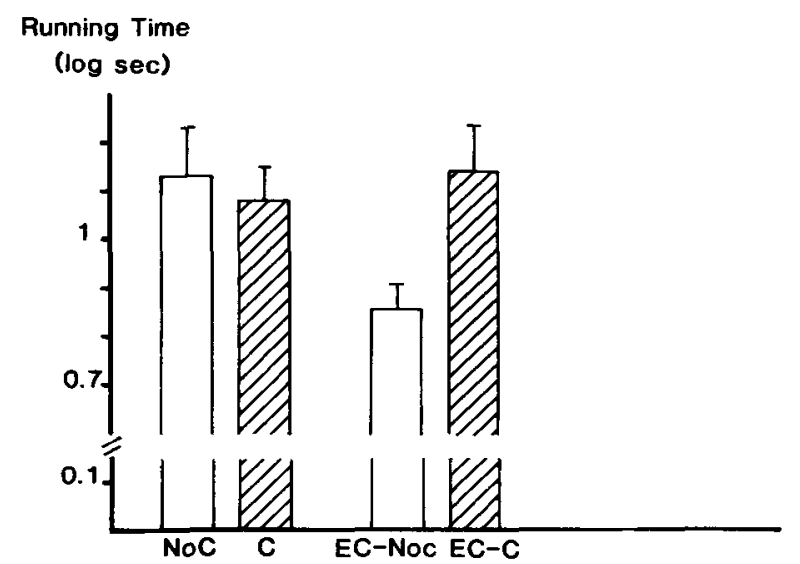

Figure 4. Mean log running time $( \pm S E M)$ during the first retention trial for each of the groups tested after the 14-day TTI. After such a retention interval, (1) there was no difference between animals tested under changed (C) or nonchanged (NoC) conditions, (2) prior exposure to the experimental context (EC) induced an improvement of performance only for nonchanged conditions (EC NoC), and (3) the effect of testing conditions could be reinstated when the retention test was preceded by an exposure to the experimental context.

after a 14-day interval there was no effect of testing conditions $[\mathrm{C}$ or NoC, $F(1,55)=1.92]$ and no effect of treatment conditions $[F(1,55)=1.88]$, but a significant interaction between treatment and testing conditions $[F(1,55)=4.12, p=.044]$.

Planned comparisons confirmed that after delayed testing, Group NoC did not differ from Group $\mathrm{C}$ during the first test trial $[F(1,26)=.20]$ or during the remaining test trials $[F(1,26)=.94]$. The same analysis revealed that the nonchanged cued animals (EC-NoC group) ex- hibited shorter running times than did the corresponding changed animals (EC-C group) $[F(1,29)=5.91$, $p=.02]$. This effect endures during the four first test trials $[F(1,29)=4.11, p=.049]$. Furthermore, the nonchanged cued animals (EC-NoC) also performed better than control animals (NoC) in terms of running time $[F(1,24)=6.11, p=.019]$; no difference could be detected between the two changed groups, C and EC-C $[F(1,25)=.81]$. This indicates that the facilitative effect of cuing on performance was obtained only for animals tested under nonchanged conditions.

\section{Discussion}

The data obtained in this experiment showed that, as expected, the mean performance of rats submitted to a long training-to-test interval (NoC 14-day) was similar to that of rats tested after 1 day in the contextual change condition (C 1-day) and that the performance of both was significantly impaired as compared with that of rats retested in the same alley after a 1-day interval (NoC 1-day).

An examination of the effect of a pretest cuing treatment on the performance of animals disrupted by a contextual change when testing took place 1 day after training indicates that none of the selected treatments was effective in alleviating the performance deficit. In our view, this lack of effect could not be attributed to an inadequate choice of the cuing treatment. Indeed, as previously noted, exposure to a part of the training apparatus or to the experimental context and the provision of access to the specific food reinforcer of the training episode are among the most widely used reactivation treatments (Deweer et al., 1980; Koppenaal et al., 1967; Sara, 1973). Moreover, the results obtained after a retention interval of 14 days demonstrate that at least one of them, exposure to the experimental context, is able to alleviate performance disruption induced by spontaneous forgetting. The latter data confirm previous findings of the facilitative effect of such a treatment on spontaneous forgetting (Deweer et al., 1980; Gatti, Pais, \& Weeks, 1975; Gisquet-Verrier et al., 1985).

The fact that none of the selected cuing treatments alleviated the performance deficit due to disruption by a contextual change, whereas one of the cuing treatments (exposure to the experimental context: EC) did alleviate the performance deficit due to an extended training-totest interval, suggests that these two disruptions of performance do not result from the same source. This makes it difficult to account for the disruption induced by contextual change in terms of retrieval failure, as has been proposed for spontaneous forgetting.

Our data clearly confirmed that after a retention interval of 14 days (see Figure 4) the contextual change no longer affected test performance. The fact that no difference could be detected between the animals tested under change or no-change conditions cannot be attributed to a ceiling effect in which forgetting of the response would obliterate the reaction to change, since, as mentioned in 
the discussion of Experiment 1, the lack of a reaction to contextual change occurred, at least in our conditions, before spontaneous forgetting of the original response.

A different picture emerged from the comparison of the two groups in which testing was preceded by an exposure to the experimental context (EC-NoC and EC-C groups), inasmuch as they differed in terms of running time during the first four testing trials. Nevertheless, we must emphasize that this difference is due merely to a facilitative effect of the cuing treatment on the performance of the no-change animals. As a matter of fact, prior exposure to the experimental context improved performance for animals tested in no-change conditions after a retention interval of 14 days, but this treatment did not have the same facilitative effect for animals tested under changed conditions.

That a pretest exposure to the experimental context differentially affected retention performance as a function of testing conditions (NoC or C) indicates that when animals no longer react to a contextual change, there is not a complete loss of the information relative to the initial pattern of the walls, as has often been postulated (Perkins \& Weyant, 1958).

\section{GENERAL DISCUSSION}

The paradox suggested by Riccio and his colleagues (Riccio \& Ebner, 1981; Riccio et al., 1984) seems to be based mainly upon the fact that performance disruptions resulting from a contextual change have been analyzed as a memory phenomenon, a case of forgetting (Gordon et al., 1981; Spear, 1978). The present experiments provide information about the contextual change effect that may be helpful in clarifying the situation.

The first experiment showed that an alteration of the context between training and testing situations, such as a change in the pattern of the walls, can dramatically affect test performance. Furthermore, in this case this contextual change had a maximal effect shortly after original training, but the effect decreased when the retention interval was lengthened. These results are consistent with previous findings related to the development of contextual generalization gradients over time, obtained after contextual changes (Perkins \& Weyant, 1958; Steinman, 1967).

Such an alleviation of the performance deficit was observed between 1 and 7 days for animals submitted to change, whereas, with the same delays, no decrement in performance was detected when the training stimuli were unchanged. Thus, the lack of a reaction to the contextual change occurs before the forgetting of the original response takes place. This confirms the similar observations of Desiderato et al. (1966) and Richardson et al. (1983). Furthermore, Experiment 2 showed that the deficit in the "changed" rats, after 1 day, was not susceptible to cuing treatments known to enhance performance in several other cases of memory disruption.

Taken together, these findings suggest that the poor test performance following contextual changes results mainly from good retention of the initial information. Obviously, the change in intrarunway context can induce a performance disruption only insofar as the subjects remember the initial situation well. So any disrupting effect of contextual changes could be conceived of as an interference: any new element in the testing situation may elicit competing orienting responses that interfere with expression of the initial response.

The second experiment showed that two deficits of performance, quantitatively equivalent but resulting either from a contextual change or from a lengthening of the retention interval (spontaneous forgetting), were differentially affected by similar treatments. None of the cuing treatments given 1 day following training alleviated performance deficits resulting from a contextual alteration. Spontaneous forgetting, on the other hand, obtained in no-change conditions after a 14-day training-to-test interval, was alleviated by exposure to the experimental context. These results, taken together, strongly suggest that disruption of performance due to contextual change cannot be analyzed, as can spontaneous forgetting, in terms of retrieval failure.

These data appear to be inconsistent with those obtained by Gordon and his colleagues (Gordon et al., 1981; Mowrer \& Gordon, 1983; Wittrup \& Gordon, 1982), who have repeatedly demonstrated that a particular cuing treatment may overcome the detrimental effect of contextual changes (change of experimental room). Nevertheless, to be effective in Gordon's paradigms, the treatment must include two successive phases: the cuing treatment itself, consisting of the 15-sec placement of the animal in a box that is identical to a part of the training apparatus, followed by a second phase in which the animal is placed, for $3.5 \mathrm{~min}$, in a holding cage in the room where the testing is to be run. These authors interpreted these results in terms of the addition of the cuing context to the training memory. But, for our purposes, these results demonstrate that for a contextual change to be efficient, the treatment has to be quite different from those used in such other cases of performance disruption as spontaneous forgetting, experimental amnesia, or the Kamin effect, paradigms within which a simple cuing procedure alleviates performance disruption. So those results do not really argue against our assertion that disruption of performance due to contextual change is not due to a retrieval deficit.

Experiment 2 provided further information concerning the contextual change effect. The absence of reactivity to delayed contextual change occurred in our conditions, whereas the running response was well maintained. The most widely accepted explanation is to consider that the animals forget the initial precise features of the stimulus on which change occurred (pattern of the walls) before they forget the target memory (i.e., the running response) (W. R. McAllister \& D. E. McAllister, 1963; Perkins \& Weyant, 1958; Riccio et al., 1984; Spear, 1978).

However, we have strong evidence that argues against such an interpretation. In fact, our results demonstrate that after a pretest reactivation of the initial memory, in 
duced by an exposure to the experimental context, animals react differently as a function of testing conditions, as they did shortly after the end of training. This indicates that, after a delayed retention test, even when contextual change no longer induces any performance disruption, animals do not completely forget the initial information. Thus, the lack of an effect of the contextual change long after training may result from a failure to retrieve the details of the training context. This finding is consistent with earlier data of Steinman (1967). In her experiment, animals were also trained to run in a straight alley in order to obtain food reinforcement. A performance deficit was obtained when testing was done in a different alley 1 day following training but not after a 66-day TTI. A second test was performed 1 day following the first retention test. This second test took place either, for half of the animals, in the same alley as for the first retention test or, for the other half, in another alley. A similar pattern of results emerged whatever the length of the initial retention interval. A performance deficit was obtained only if the retest was the first exposure to the novel alley (AAB). On the other hand, subjects shifted from the novel test alley back to the initial alley (ABA) performed as did animals retested in the same test alley (ABB) or as did animals that had never been changed (AAA). So, even 67 days after training, the initial alley was not considered as a novel alley. These results provide "evidence that the stimulus conditions originally employed were not completely lost over time" (Steinman, 1967, p. 108).

It has often been proposed that pretest cuing reactivates "forgotten" memories and brings them to a state that is similar to that of initial training, defined by Lewis (1979) as an "active memory." Numerous experiments have already demonstrated that after a previous reactivation, an old memory becomes susceptible again either to amnestic treatments (Misanin, Miller, \& Lewis, 1968; see also Spear, Hamberg, \& Bryan, 1980, and Riccio \& Richardson, 1984) or to facilitatory treatments (De Vietti, Conger, \& Kirkpatrick, 1977). The present studies provide further information concerning the concept of active memory by demonstrating that an old reactivated memory is equally vulnerable to a contextual change.

It has been well established that impaired retention performance due to extended TTI does not result from the loss of a part of the target memory but to an inability to retrieve correctly the initial information. Our data show that, similarly, nonreaction to a contextual change does not imply a loss of the corresponding contextual attribute but, rather, is due to an impaired retrieval of details concerning the training context.

Apart from these assertions, the experimental data show that a contextual change might induce poor test performance even after a long training-to-test interval. They support the hypothesis that cuing treatment produces reactivation not only of the target memory, but also of other memory attributes, such as those representing the pattern of the walls.
The principal aim of this paper was to shed some light on the causes of the paradox identified by Riccio and his colleagues (Riccio et al., 1984): "If changes in stimulus characteristics become less disruptive with delayed testing, then how can subtle changes in context provide the basis for failure to retrieve a response?' (p. 161). In our view, this paradox, which is an embarrassment for the contextual theory of forgetting formulated by Spear (1978), may be explained by a confusion between different phenomena falsely considered to be equivalent. One source of such confusion may derive from Spear's definition of forgetting in which the term is used in a strictly operational sense: " 'forgetting' caused by a specified event is defined by a decrement relative to the same index assessed when that even is absent' (p. 7). In other words, according to this definition, the performance disruptions resulting either from a contextual change or from an extended training-to-test interval can both be considered as two typical cases of "forgetting." Nevertheless, even if these two performance deficits can be operationally labeled as "forgetting," their sources may not necessarily be the same. In fact, the present experiments have demonstrated that a prior cuing treatment may alleviate a performance deficit resulting from an extended training-totest interval but not one resulting from a contextual change introduced shortly after training. These results suggest that only the former performance deficit can be considered as a memory-retrieval failure. On the other hand, as we have already pointed out, the disruption induced by contextual change must actually be due to a good retention of the initial information.

The second source of confusion is due to the fact that the change in context that leads to the well-documented contextual change effect is hypothetically assimilated to changes that inevitably occur during the training-to-test interval. The difference between these two kinds of changes is obvious from a descriptive point of view as well as from the experimental data of this study: (1) The nature of the contextual changes involved in both situations is different. In the first case, contextual change is explicit, introduced by the experimenter, whereas in the second case, forgetting is considered as being due to a progressive accumulation of subtle and implicit changes taking place during the retention interval (Spear, 1978). (2) The fact that the same cuing treatments do not have the same efficacy when delivered in each of the two kinds of contextual changes and the fact that the disruption induced by one of the contextual changes decreases over time while the other increases constitute further support for the notion that the contextual changes implicated in both situations differ radically.

In light of the evidence presented here, the paradox appears to result from a syllogism, which can roughly be expressed in the following terms: Contextual changes induce a disruption of performance, operationally labeled as forgetting (major premise); the longer the training-totest interval, the greater the contextual changes (minor 
premise); hence, spontaneous forgetting is determined by the increase of contextual changes (conclusion). Such a syllogism must, actually, be considered as a sophism, since it is based upon a dual confusion relative to the definition of forgetting and to different kinds of contextual changes, as pointed out above.

In fact, Riccio and his colleagues (Riccio \& Ebner, 1981; Riccio et al., 1984) not only called attention to the circularity involved in the "contextual cues model" but also proposed new ways of thinking about the nature of forgetting. They suggested that the generalization phenomenon, which increasingly affects the training stimuli, "could provide a plausible mechanism by which interference, extinction, reencoding and so on, might impair memory retrieval'' (Riccio et al., 1984, p. 163).

This paper was not aimed at clearly defining to what extent numerous subtle contextual changes that inevitably occur during the retention interval participate in the general phenomenon of forgetting. Nevertheless, even if implicated, these changes do not affect test performance in the same way as does a manipulated contextual change introduced shortly after training.

The major point is that, without a doubt, similarity between training and testing context determines good performance. But, even so, it should not be inferred that forgetting, as assessed by poor test performance, is mainly due to dissimilarity between these two situations. In the same way, enhancement of retention performance by prior cuing does not necessarily imply that cuing enhances similarity between training and testing situations. These assertions do not rule out the importance of contextual alterations in memory processes; they only limit it somewhat.

\section{REFERENCES}

Desiderato, O., Butler, B., \& Meyer, C. (1966). Changes in fear generalization gradients as a function of delayed testing. Journal of Experimental Psychology, 72, 678-682.

DE Vietti, T. L., Conger, G. L., \& KirkPatrick, B. R. (1977). Comparison of the enhancement gradients of retention obtained with stimulation of the mesencephalic reticular formation after training or memory reactivation. Physiology \& Behavior, 19, 549-554.

DeWEer, B., SARA, S. J. (1984). Background stimuli as a reminder after spontaneous forgetting: Role of duration of cuing and cuing-test interval. Animal Learning \& Behavior, 12, 238-247.

Deweer, B., Sara, S. J., \& Hars, B. (1980). Contextual cues and memory retrieval in rats: Alleviation of forgetting by pretest exposure to background stimuli. Animal Learning \& Behavior, 8, 265-272.

GatTI, S. V., PAIS, N., WeEks, J. R. (1975). Effect of reinstatement procedure on retention of differential appetitive responding. Bulletin of the Psychonomic Society, 6, 57-60.

GISQUET-VERRIER, P. (1981). Accelerated extinction after post-trial halothane anaesthesia in rats: An aversive effect. Physiology \& Behavior, 26, 223-231.

Gisquet-Verrier, P., Dekeyne, A., \& Alexinsky, T. (1985). Memory reorganization over time as revealed by interaction between type of pre-test cueing and length of retention interval. In B. E. Will, P. Schmitt, \& J. C. Dalrymple-Alford (Eds.), Brain plasticity, learning and memory. New York and London: Plenum Press.
Gordon, W. C., McCracken, K. M., Dess-Beech, N., * Mowrer, R. R. (1981). Mechanisms for the cueing phenomenon: The addition of the cueing context to the training memory. Learning \& Motivation, 12, 196-211.

HollowaY, F. A., \& WANSLEY, R. (1973). Multiphasic retention deficits at periodic intervals after passive avoidance learning. Science, 180, 208-210.

HUPPERT, F. A., \& DEUTSCH, J. A. (1969). Improvement in memory with time. Quarterly Joumal of Experimental Psychology, 21, 267-271.

KePPEL, G. (1972). Forgetting. In L. Sechrist, C. P. Duncan, \& A. W. Melton (Eds.), Festschrift for Benton J. Underwood. New York: Appleton-Century-Crofts.

KuEIN, S. B., \& SPEAR, N. E. (1970). Reactivation of avoidance-learning memory in the rat after intermediate intervals. Journal of Comparative Physiological Psychology, 72, 498-504.

KoppenaAl, R. J., Jagoda, E., CRUCE, J. A. F. (1967). Recovery from ECS-produced amnesia following a reminder. Psychonomic Science, 9, 293-294.

LEWIS, D. J. (1979). Psychobiology of active and inactive memory. Psychological Bulletin, 86, 1054-1083.

MaDGsick, W. K. (1936). The curve of retention of an incompletely learned problem in albino rats at various age levels. Journal of Psychology, 2, 25-48.

MCAllister, W. R., \& MCAllister, D. E. (1963). Increase over time in the stimulus generalization of acquired fear. Joumal of Experimental Psychology, 65, 576-582.

Misanin, J. R., Miller, R. R., \& Lewis, D. J. (1968). Retrograde amnesia produced by electroconvulsive shock after reactivation of a consolidated memory trace. Science, 160, 554-555.

MOWRER, R. R., \& Gordon, W. C. (1983). Effect of cueing in an "irrelevant" context. Animal Learning \& Behavior, 11, 401-406.

OVERTON, D. (1964). State-dependent or "dissociated" learning produced with pentobarbital. Journal of Comparative Physiological Psychology, 57, 3-12.

Perkins, C. C., \& Weyant, R. G. (1958). The interval between training and test trial as determiner of the slope of generalization gradients. Journal of Comparative \& Physiological Psychology, 51, 596-600.

Pesselman, M. L., \& Riccio, D. C. (1980, April). Forgetting of stimulus attributes of a fear stimulus. Paper presented at the meeting of the Eastern Psychological Association, Hartford, CT.

Postman, L. (1971). Transfer, interference and forgetting. In J. W. Kling \& L. A. Riggs (Eds.), Woodworth and Schlosberg's experimental psychology. New York: Holt, Rinehart \& Winston.

Riccio, D. C., \& EBNer, D. L. (1981). Post-acquisition modifications of memory. In N. E. Spear \& R. R. Miller (Eds.), Information processing in animals, memory mechanisms (pp. 291-317). Hillsdale, NJ: Erlbaum.

Riccio, D. C., Richardson, R. (1984). The status of memory following experimentally induced amnesias: Gone but not forgotten. Physiological Psychology, 12, 59-72.

Riccio, D. C., Richardson, R., \& EbNer, D. L. (1984). Memory retrieval deficits based upon altered contextual cues: A paradox. Psychological Bulletin, 96, 152-165.

Richardoson, R., Riccio, D. C., Guanowsky, V., \& OKonkwo, A. E. (1983, May). Disruptive effects of context shifts diminish as a function of retention interval. Paper presented at the meeting of the Midwestern Psychological Association, Chicago.

SARA, S. J. (1973). Progressive development of avoidance responses after training, ECS and repeated testing. Bulletin of the Psychonomic Society, 2, 134-136.

Seybert, J. A., Wilson, M. A., \& ARcher, A. L. (1982). The Kamin effect as a function of training and associative-nonassociative processes. Bulletin of the Psychonomic Society, 19, 227-230.

Smith, S. M., Glenburg, A., \& BjorK, R. A. (1978). Environmental context and human memory. Memory \& Cognition, 6, 342-353.

SPEAR, N. E. (1973). Retrieval of memory in animals. Psychological Review, 80, 163-194.

SPEAR, N. E. (1978). The processing of memories: Forgetting and retention. Hillsdale, NJ: Erlbaum. 
Spear, N. E., Gordon, W. C., \& Martin, P. A. (1973). Warm up decrement as failure in memory retrieval in the rat. Journal of Comparative \& Physiological Psychology, 85, 601-614.

Spear, N. E., Hamberg, J. M., \& Bryan, R. (1980). Forgetting of recently acquired or recently reactivated memories. Learning \& Motivation, $11,456-475$.

Steinman, F. (1967). Retention of alley brightness in the rat. Journal of Comparative \& Physiological Psychology, 64, 104-109.
Tulving, E., \& Thомson, D. (1973). Encoding specificity and retrieval processes in episodic memory. Psychological Review, 80, 352-373. WitTruP, M., Gordon, W. C. (1982). Alteration of a training memory through cueing. American Journal of Psychology, 95, 495-507.

(Manuscript received February 4, 1986; revision accepted for publication June 17, 1986.) 